\title{
Shape Recognition Using V4 Dominant Conical Meshes
}

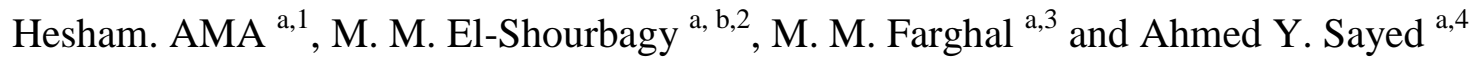 \\ a- Department of Physics \& Engineering Mathematics, Faculty of Engineering El-Mataria, Helwan University, \\ Cairo, Egypt \\ b- Higher Institute of Engineering, El-Shorouk Academy, El-Shorouk City, Cairo, Egypt. \\ (pdeham@m-eng.helwan.edu.eg) a, 1, (moatasem_alshurbagy@m-eng.helwan.edu.eg) a, b, 2 , \\ (m.m.farghal86@gmail.com) ${ }^{\text {a, } 3}$, (AHMED_BADR@m-eng.helwan.edu.eg) a, 4
}

\begin{abstract}
This research work introduces a new concept of shape recognition where we obtain a geometric form from some given parameters related to architecture geometry. They include cell face slope, edge length initial value of the vertical alignment and boundary vertices of the horizontal alignment. Here we construct a conical mesh with horizontal planar latitudes from initial horizontal and vertical alignments. For this purpose, analytic geometry, interpolation, data structures using Visual C++ Programming, computational geometry and CAD features are used.
\end{abstract}

Keywords: Architectural geometry, computer aided geometric modelling, shape recognition, conical meshes, interpolation and computational geometry.

\subsection{Introduction}

Conical meshes were presented by [1] as shown in Fig. 1. It was proved that a mesh valence 4 vertex is conical if and only if:

$$
\omega_{1}+\omega_{3}=\omega_{2}+\omega_{4}
$$

A description of meshes that admit uniform distance edge offsets based on a graphtheoretic analysis of the underlying dual mesh was presented by [2] as shown in Fig. 2. The use of ruled surfaces for geometric modelling or approximation was investigated by [3] as shown in Fig. 3. The concept of geometry from combinatory was introduced by [4] as shown in Fig. 4. 


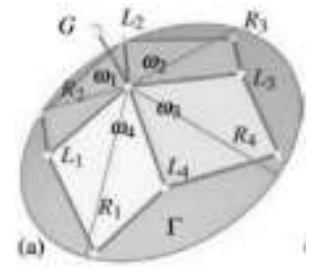

Fig. 1: Four faces touch a common cone $\Gamma$ and have interior angles $\omega_{1}, \ldots, \omega_{4}$.

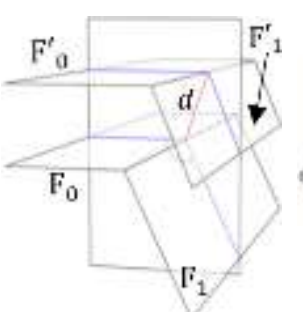

Fig. 2: Plane generated by normals of $F_{0}, F_{1}$.

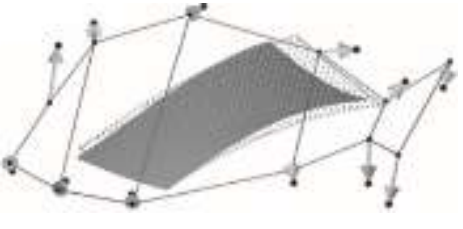

Fig. 3: The control points of a ruled B-spline surface are displaced to optimally approximate a given point cloud.

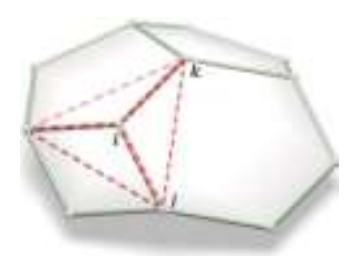

Fig. 4: A mixed polygonal mesh. The vertex star around vertex $i$ consists of all triangles generated by the edges through $i$.

\subsection{Overview}

Planar quad meshes have many advantages stated in [5] and [6]. Thus, we aim to implement a conical valence 4 dominant mesh depending on a closed discrete planar curve as an initial horizontal alignment. The opened discrete planar curve is an initial vertical alignment. In the next section we introduce a geometric modelling approach. Section 3 shows some theorems for constructing different forms of horizontal and vertical alignments. Section 4 shows an algorithm of constructing a conical mesh. Section 5 shows some implemented algorithms results. Section 6 shows some geometric analysis. Section 7 shows the discussion, while section 8 concludes our research work.

\section{Geometric Modelling Approach}

\subsection{Cells Semi Conical Angle}

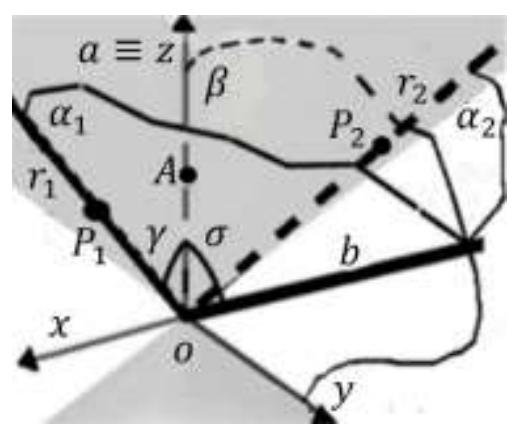

Fig. 5: The conical angle is the dihedral angle $\left(P_{1}, b, P_{2}\right)$. The semi conical angle is the dihedral angle $\left(P_{1}, b, A\right)$.

Theorem 1: For a right circular cone with semi vertex angle $\gamma$ and axis $a$, if $\alpha_{1}, \alpha_{2}$ are two tangent planes to the cone at $r_{1}, r_{2}, P_{1} \in r_{1}, P_{2} \in r_{2}, b\left(\alpha_{1} \cap \alpha_{2}\right)$, plane $\beta[a, b]$ and $\sigma=\varangle(a, b)$ i.e. $\gamma<\sigma<\pi-\gamma$. Then, the semi conical angle $\delta=$ $\Varangle\left(P_{1}, b, A\right)$ will be given by the relation:

$$
\sin \delta=\frac{\sin \gamma}{\sin \sigma}
$$


The conical angle will be given by the relation:

$$
\cos 2 \delta=1-2\left(\sin ^{2} \gamma\right)\left(\csc ^{2} \sigma\right)
$$

Proof: For simplicity let the vertex be at origin, $a \equiv z$-axis and $\beta \equiv$ plane $y z$ as shown in Fig. 5.

$$
\text { Let } P_{1}\left(x_{1}, y_{1}, z_{1}\right) . \quad \therefore x_{1}{ }^{2}+y_{1}{ }^{2}=z_{1}{ }^{2} \tan ^{2} \gamma .
$$

Also

The gradient vector $\vec{d}\left\langle x_{1}, y_{1},-z_{1} \tan ^{2} \gamma\right\rangle$ is a normal vector of $\alpha_{1}$.

$\because \vec{e}\langle 0, \sin \sigma, \cos \sigma\rangle$ is a unit vector in the direction of $b$ and $\vec{d} \perp \vec{e}$.

$\therefore$ Based on the result of dot product of the orthogonal vectors, one can conclude that:

$$
y_{1}=z_{1}\left(\tan ^{2} \gamma\right)(\cot \sigma)
$$

From $(a)$ and $(b)$ we get: $x_{1}= \pm z_{1}(\tan \gamma) \sqrt{1-\left(\tan ^{2} \gamma\right)\left(\cot ^{2} \sigma\right)}$.

$\therefore \overrightarrow{\mathrm{d}}=\mathrm{z}_{1}(\tan \gamma)\left\langle \pm \sqrt{1-\left(\tan ^{2} \gamma\right)\left(\cot ^{2} \sigma\right)},(\tan \gamma)(\cot \sigma),-\tan \gamma\right\rangle$

$\because \vec{f}\langle 1,0,0\rangle$ is a normal vector of $\beta$.

$$
\therefore \cos \delta=(\cos \gamma) \sqrt{1-\left(\tan ^{2} \gamma\right)\left(\cot ^{2} \sigma\right)}, \quad \cos \gamma>0,
$$

$\therefore \sin \delta=\sqrt{1-\cos ^{2} \delta}=\frac{\sin \gamma}{\sin \sigma}$

$\therefore \cos 2 \delta=\cos ^{2} \delta-\sin ^{2} \delta=1-2\left(\sin ^{2} \gamma\right)\left(\csc ^{2} \sigma\right) \mathbf{m}$.

\subsection{Valence 3 Conical Vertex}

Theorem (2): Any mesh valence 3 vertex is conical.

Proof: Let $a$ be a line with direction cosines $l, m, n$ making the same angle of inclination on the three planes $\alpha_{1}, \alpha_{2}, \alpha_{3}$ as shown in Fig. 6. Let $\left\langle E_{i}, F_{i}, G_{i}\right\rangle$ be normal vector components of $\alpha_{\mathrm{i}}$ where $i=1,2,3$. Assume $g_{i}=\sqrt{\left(E_{i}\right)^{2}+\left(F_{i}\right)^{2}+\left(G_{i}\right)^{2}}$.

$\therefore \frac{\left|l E_{1}+m F_{1}+n G_{1}\right|}{g_{1}}=\frac{\left|l E_{2}+m F_{2}+n G_{2}\right|}{g_{2}}=\frac{\left|l E_{3}+m F_{3}+n G_{3}\right|}{g_{3}}$

Assuming:

$$
\left.\begin{array}{r}
\left\{\begin{array}{l}
d_{1}=\left(F_{1} g_{3}-F_{3} g_{1}\right)\left(G_{1} g_{2}-G_{2} g_{1}\right)-\left(F_{1} g_{2}-F_{2} g_{1}\right)\left(G_{1} g_{3}-G_{3} g_{1}\right) \\
e_{1}=\left(E_{1} g_{2}-E_{2} g_{1}\right)\left(G_{1} g_{3}-G_{3} g_{1}\right)-\left(E_{1} g_{3}-E_{3} g_{1}\right)\left(G_{1} g_{2}-G_{2} g_{1}\right)
\end{array}\right. \\
f_{1}=\left(E_{1} g_{3}-E_{3} g_{1}\right)\left(F_{1} g_{2}-F_{2} g_{1}\right)-\left(E_{1} g_{2}-E_{2} g_{1}\right)\left(F_{1} g_{3}-F_{3} g_{1}\right)
\end{array}\right\}
$$




$$
\begin{aligned}
& \left\{\begin{array}{l}
d_{3}=\left(F_{1} g_{3}+F_{3} g_{1}\right)\left(G_{1} g_{2}-G_{2} g_{1}\right)-\left(F_{1} g_{2}-F_{2} g_{1}\right)\left(G_{1} g_{3}+G_{3} g_{1}\right) \\
e_{3}=\left(E_{1} g_{2}-E_{2} g_{1}\right)\left(G_{1} g_{3}+G_{3} g_{1}\right)-\left(E_{1} g_{3}+E_{3} g_{1}\right)\left(G_{1} g_{2}-G_{2} g_{1}\right) \\
f_{3}=\left(E_{1} g_{3}+E_{3} g_{1}\right)\left(F_{1} g_{2}-F_{2} g_{1}\right)-\left(E_{1} g_{2}-E_{2} g_{1}\right)\left(F_{1} g_{3}+F_{3} g_{1}\right)
\end{array}\right. \\
& \left\{\begin{array}{l}
d_{4}=\left(F_{1} g_{3}+F_{3} g_{1}\right)\left(G_{1} g_{2}+G_{2} g_{1}\right)-\left(F_{1} g_{2}+F_{2} g_{1}\right)\left(G_{1} g_{3}+G_{3} g_{1}\right) \\
e_{4}=\left(E_{1} g_{2}+E_{2} g_{1}\right)\left(G_{1} g_{3}+G_{3} g_{1}\right)-\left(E_{1} g_{3}+E_{3} g_{1}\right)\left(G_{1} g_{2}+G_{2} g_{1}\right) \\
f_{4}=\left(E_{1} g_{3}+E_{3} g_{1}\right)\left(F_{1} g_{2}+F_{2} g_{1}\right)-\left(E_{1} g_{2}+E_{2} g_{1}\right)\left(F_{1} g_{3}+F_{3} g_{1}\right)
\end{array}\right. \\
& \therefore m=n\left[\frac{e_{j}}{f_{j}}\right], l=n\left[\frac{d_{j}}{f_{j}}\right] \text { where } j=1, \ldots, 4 \therefore d_{j}, e_{j}, f_{j} \text { are direction ratios of } a_{j} .
\end{aligned}
$$

Hence, there are four cones touching the three planes $\alpha_{1}, \alpha_{2}, \alpha_{3}$

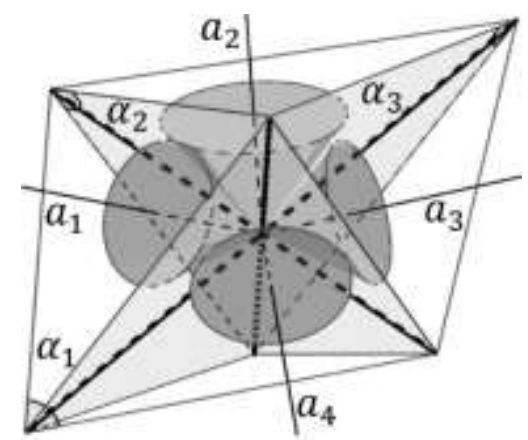

Fig. 6: Three planes $\alpha_{1}, \alpha_{2}$ and $\alpha_{3}$ intersecting at $V$ have four cones of revolution whose common vertex is $V$ and axes $a_{1}, \ldots, a_{4}$. Each cone touches the three planes.

Example: Find the direction ratios of the axes of the cones touching the three planes whose normal vectors: $\langle 1,1,1\rangle,\langle 1,-2,2\rangle,\langle-2,0,1\rangle$.

Solution: from above (a), (b), (c), (d), the direction ratios of the four axes are given

$\left\{\begin{array}{c}d_{1}=-4.3 \\ e_{1}=3.28 \\ f_{1}=34.01\end{array},\left\{\begin{array}{c}d_{2}=14.69 \\ e_{2}=-34.46 \\ f_{2}=-13.22\end{array},\left\{\begin{array}{c}d_{3}=-26.69 \\ e_{3}=4.46 \\ f_{3}=-10.77\end{array},\left\{\begin{array}{c}d_{4}=16.29 \\ e_{4}=26.72 \\ f_{4}=-10.01\end{array}\right.\right.\right.\right.$

by:

Theorem (3): If two adjacent planes of each row of valence 4 mesh with horizontal latitudes have equal slopes, their common meridian will be a vertical plane. Each mesh vertex will be conical one.

Proof: let $r_{i, j}^{\prime}$ be the horizontal projection of $r_{i, j}\left(\alpha_{i, j} \cap \alpha_{i, j-1}\right)$ as shown in Fig. 7.

$$
\because \varangle\left(\alpha_{i, j}, \pi_{i}\right)=\Varangle\left(\alpha_{i, j-1}, \pi_{i}\right) . \therefore r_{i, j}^{\prime} \text { bisects } \Varangle\left(l_{i, j}, l_{i, j-1}\right) \text {. }
$$

Similarly: $r^{\prime}{ }_{i+1, j}$ bisects $\Varangle\left(l^{\prime}{ }_{i+1, j}, l^{\prime}{ }_{i+1, j-1}\right)$. But $l_{i, j} / / l_{i+1, j}, l_{i, j-1} / / l_{i+1, j-1}$.

$$
\therefore r_{i, j}^{\prime} \equiv r_{i+1, j}^{\prime} \equiv r_{j}^{\prime} \text { which is the horizontal edge view of the meridian }
$$

For a vertex $v_{i+1, j}, \omega_{1}=\omega_{3}, \omega_{2}=\omega_{4} \because \omega_{1}+\omega_{3}=\omega_{2}+\omega_{4} \therefore$ it is a special case of a conical vertex 


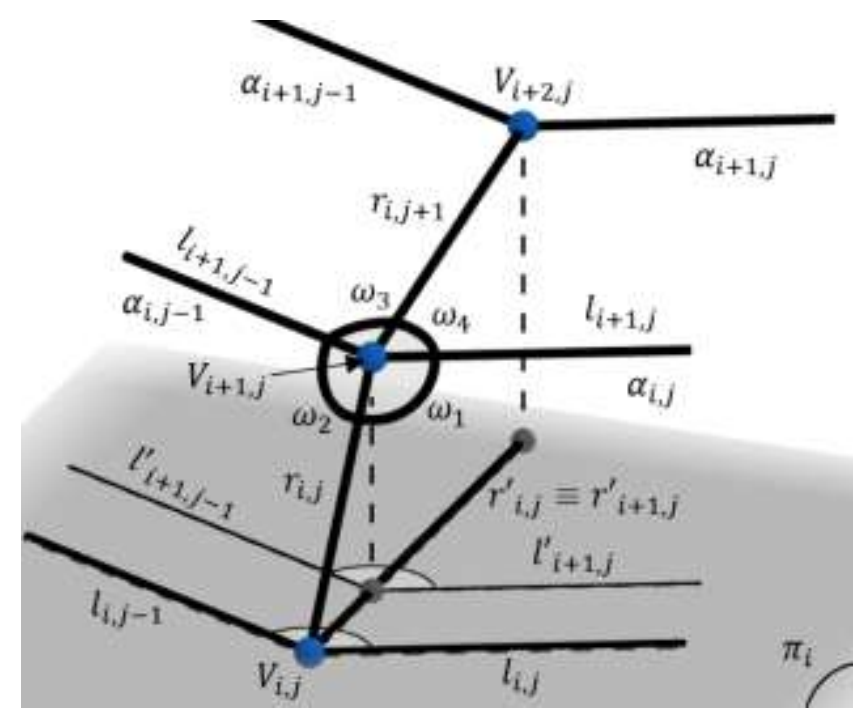

Fig. 7: A special case of a conical vertex where $\omega_{1}=\omega_{3}, \omega_{2}=\omega_{4}$.

\section{Alignment Geometric Construction}

\subsection{Elliptic Horizontal Alignment}

Theorem (4): If the given mesh boundary is an ellipse discretized by taking $n-1$ points in each quadrant, each is given by $r\left(\theta_{i}\right)$ where $\theta_{i+1}=\left(\theta_{i}+\frac{\pi}{2 n}\right)$. The length of the chord (boundary edge) increases gradually from the vertex to the co-vertex. This division suits the nature of curvature which decreases from the vertex to the co-vertex.

Proof: A point on an ellipse is given by $\left(h+a \cos \theta_{i}, k+b \sin \theta_{i}\right)$ as shown in Fig 8 .

$\therefore$ The distance between any two successive points is given by:

$$
L_{i}=\sqrt{a^{2}\left(\cos \theta_{i+1}-\cos \theta_{i}\right)^{2}+b^{2}\left(\sin \theta_{i+1}-\sin \theta_{i}\right)^{2}}
$$

$\cos \theta_{i+1}-\cos \theta_{i}=\cos \left(\theta_{i}+\frac{\pi}{2 n}\right)-\cos \theta_{i}=\left(\cos \theta_{i}\right)\left(\left(\cos \frac{\pi}{2 n}\right)-1\right)-\sin \theta_{i} \sin \frac{\pi}{2 n}=$ $-2 \cos \theta_{i} \sin ^{2} \frac{\pi}{4 n}-2 \sin \theta_{i} \sin \frac{\pi}{4 n} \cos \frac{\pi}{4 n}=-2 \sin \frac{\pi}{4 n}\left[\cos \theta_{i} \sin \frac{\pi}{4 n}+\sin \theta_{i} \cos \frac{\pi}{4 n}\right]=$ $-2 \sin \frac{\pi}{4 n} \sin \left(\theta_{i}+\frac{\pi}{4 n}\right)$.

Similarly $\sin \theta_{i+1}-\sin \theta_{i}=2 \sin \frac{\pi}{4 n} \cos \left(\theta_{i}+\frac{\pi}{4 n}\right)$

Hence $L_{i}=\sqrt{4 a^{2} \sin ^{2} \frac{\pi}{4 n} \sin ^{2}\left(\theta_{i}+\frac{\pi}{4 n}\right)+4 b^{2} \sin ^{2} \frac{\pi}{4 n} \cos ^{2}\left(\theta_{i}+\frac{\pi}{4 n}\right)}=$ $2 \sin \frac{\pi}{4 n} \sqrt{\left(a^{2}-b^{2}\right) \sin ^{2}\left(\theta_{i}+\frac{\pi}{4 n}\right)+b^{2}}$ where $a, b, n$ are constants, $a>b$. 

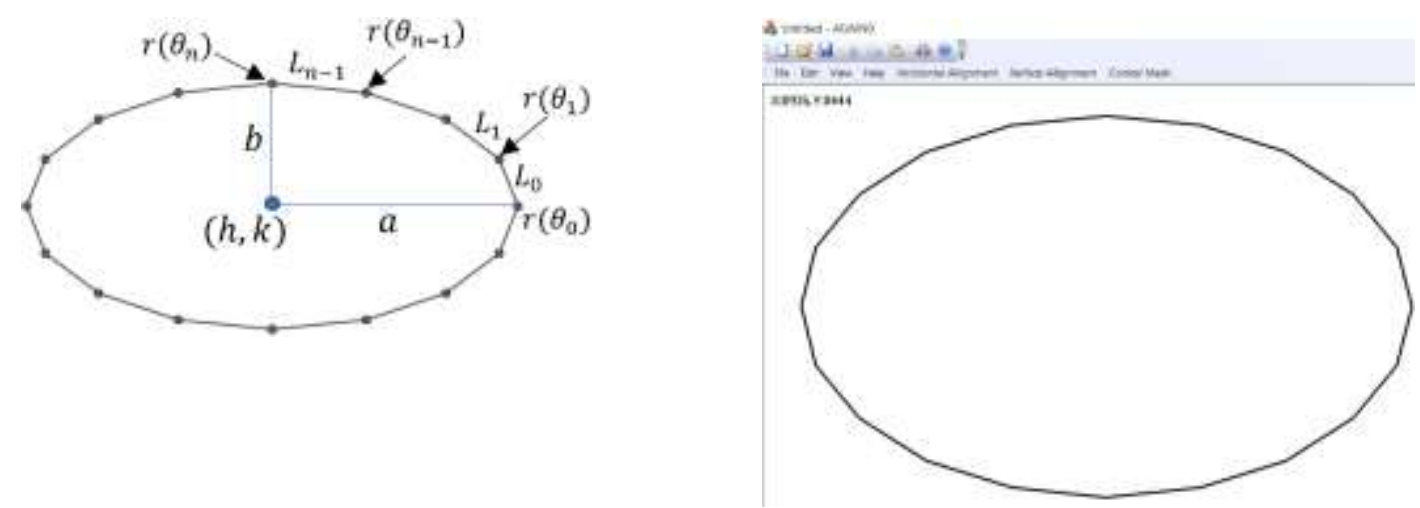

Fig. 8: A discrete analog of an ellipse centered at $(h, k)$ and whose axes $2 a$ and $2 b$. The length

Fig. 9: An output screen for a dicrete analog of an ellipse. of the chord increases gradually from the vertex to the covertex.

\section{//function for determining points on an ellipse}

void ellipHor(double a, double b, double theta, double *xEllip, double *yEllip)\{

double thetaRad $=$ theta $* 3.14159265358979 / 180$;

$*$ xEllip $=-\mathrm{a} *(\cos ($ thetaRad $))+550$;

$*$ yEllip $=b *(\sin ($ thetaRad $))+350 ;\}$

See the output screen in Fig. 9.

\subsection{Given Boundary Vertices Interpolation}

Given a number of boundary points, Newton Forward divided difference interpolation is used to construct a closed curve as a union of two polynomial curved segments of different degrees. The resulted closed curve is divided into any required number of vertices.

Theorem (5): If a parabolic curved segment with vertical axis and vertex $V\left(x_{0}, y_{0}\right)$, is discretized by taking n points $P_{i}\left(x_{I}, y_{I}\right)$ to the right (or left) of $V$ where $x_{i+1}=x_{i}+h$ where $h=\frac{x_{n}-x_{0}}{n}$. The length of a chord increases gradually from $\mathrm{V}$ to $\mathrm{P}_{\mathrm{n}}$. This division suits the nature of curvature at the curve vertex.

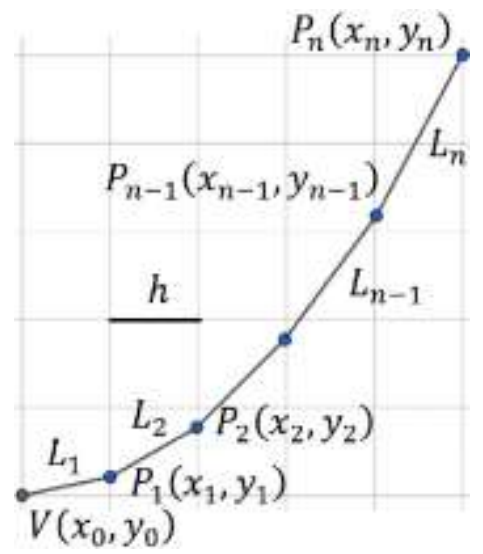

Fig. 10: A discrete analog of a parabolic segment. The length of the chord increases gradually from the vertex. 
Proof: Given a parabolic curved segment with vertical axis as shown in Fig 10. The curve is given by: $y=a x^{2}+b x+c$. As $x_{0}=\frac{-b}{2 a}$. Then $x_{i}=\frac{-b}{2 a}+h i$. Hence, the length of any chord $\overline{P_{l}, P_{l+1}}$ is given by: $L_{i}=h \sqrt{1+h^{2} a^{2}(2 i+1)^{2}}$, where $a, h$ are constants $\therefore L_{i}$ increases as $|i|$ increases which suits the nature of curvature at the curve vertex $\boldsymbol{\square}$.

\section{An Algorithm for Given Boundary Shape Recognition with Conical}

\section{Meshes}

Let $V_{0,0}, V_{0,1}, \ldots, V_{0, n-1}$ be planar polygon vertices in the plane $E^{2}$. One can consider it as an initial part of the horizontal alignment of a conical mesh in $E^{3}$. These polygons are called the latitudes. The 2D mesh will be considered as a 3D surface has also an initial vertical alignment which is a planar discrete analog of a curve $V_{0,0}, V_{1,0}, \ldots, V_{m-1,0}$. These curves are called meridians. Each latitude is a horizontal planar closed discrete analog of a curve whose plane $\pi_{i}$ equation is given by $z=V_{i, 0}$.

\subsection{A Brief Outline Of The Algorithm}

The following algorithm constructs a row $i$ of the valence 4 dominant conical mesh. The row lies between the two horizontal planes $\pi_{i}, \pi_{i+1}$.

1 - Construct the plane $\alpha_{i, 0}\left(V_{i, 0}, V_{i, 1}, V_{i+1,0}\right)$ as shown in Fig 11. Calculate $\theta_{i}=$ Z $\left(\alpha_{i, 0}, \pi_{i}\right)$.

2- Construct planes $\alpha_{\mathrm{i}, \mathrm{j}} \supset\left\{\mathrm{V}_{\mathrm{i}, \mathrm{j}}, \mathrm{V}_{\mathrm{i}, \mathrm{j}+1}\right\}$, where $\Varangle\left(\alpha_{i, j}, \pi_{i}\right)=\theta_{i}, j=1,2, \ldots, n-1$ as shown in Fig 12.

3-Compute intersection lines $r_{i, j}\left(\alpha_{i, j-1} \cap \alpha_{i, j}\right), t_{i, j}\left(\alpha_{i, j-1} \cap \alpha_{i, j+1}\right)$ and $s_{i, j}\left(\alpha_{i, j-1} \cap \alpha_{i, j+2}\right)$ where their intersections with plane $\pi_{i+1}$ are the points $V_{i+1, j}^{\prime}, T_{i+1, j}^{\prime}$ and $S_{i+1, j}^{\prime}$ respectively as shown in Fig. 13.

4- If $T_{i, j}\left(r_{i, j} \cap r_{i, j+1}\right) \in \overline{V_{l, j}, V_{l+1, j}^{\prime}}$; join $\overline{V_{l, j} T_{l, j}}, \overline{V_{l, j+1} T_{l, j}}$.

a- If $S_{i, j}\left(t_{i, j} \cap r_{i, j+2}\right) \in \overline{T_{l, j}, T_{l+1, j}^{\prime}}$ : join $\overline{T_{l, j} S_{l, j}}, \overline{V_{l, j+2} S_{l, j}}$.

If $U_{i, j}\left(s_{i, j} \cap r_{i, j-1}\right) \in \overline{S_{l, j}, S_{l+1, j}^{\prime}}$ : join $\overline{S_{l, j} U_{l, j}}, \overline{V_{l, j-1} U_{l, j}}$ and $\overline{U_{l, j} V_{l+1, j}}$ as shown in Fig. 14.

Otherwise, $V_{i+1, j} \equiv S_{i+1, j}^{\prime}$, join $\overline{S_{l, J} S_{l+1, j}^{\prime}}$.

b- Otherwise: $V_{i+1, j} \equiv T_{i+1, j}^{\prime}$, join $\overline{T_{l, j} V_{l+1, j}}$.

Otherwise, $V_{i+1, j} \equiv V_{i+1, j}^{\prime}$, join $\overline{V_{l, j} V_{l+1, j}}$ 


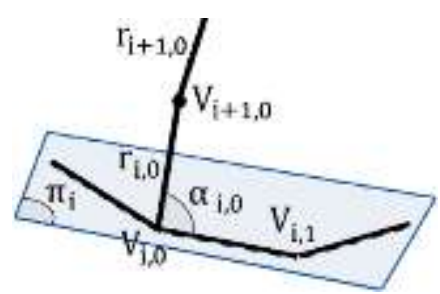

Fig. 11: Construction of the first plane in the row.

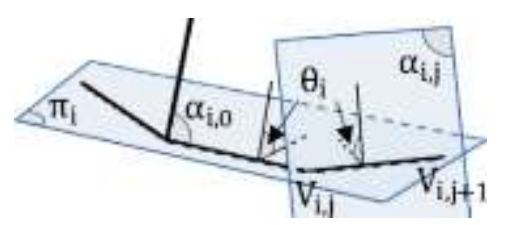

Fig. 12: Construction of the general plane in the row.

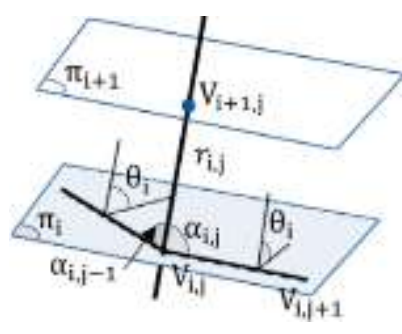

Fig. 13: Construction of the line $r_{i, j}$ and the point $V_{i+1, j}$.

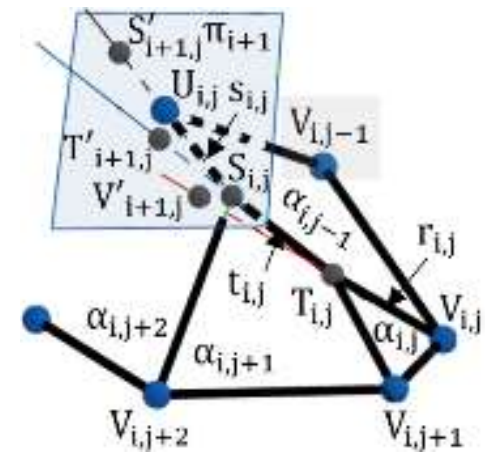

Fig. 14: Points $T_{i, j}, S_{i, j}, U_{i, j}, T^{\prime}{ }_{i+1, j}, S^{\prime}{ }_{i+1, j}$, $U^{\prime}{ }_{i+1, j}$ and lines $t_{i, j}, s_{i, j}$

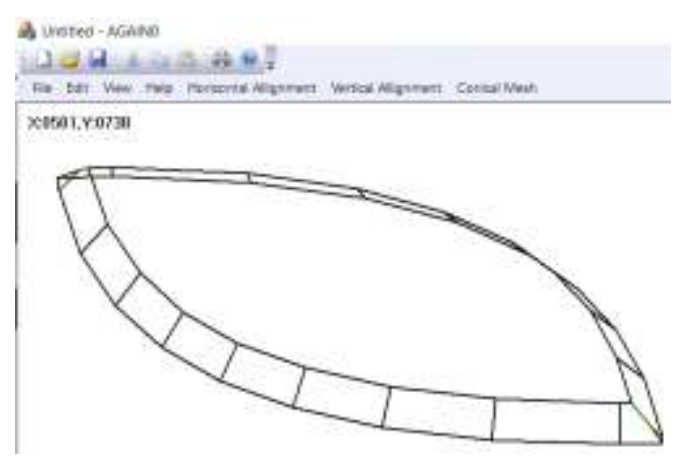

Fig. 15: An output screen for one row in Axonometric projection.

\subsubsection{Algorithm Implementation}

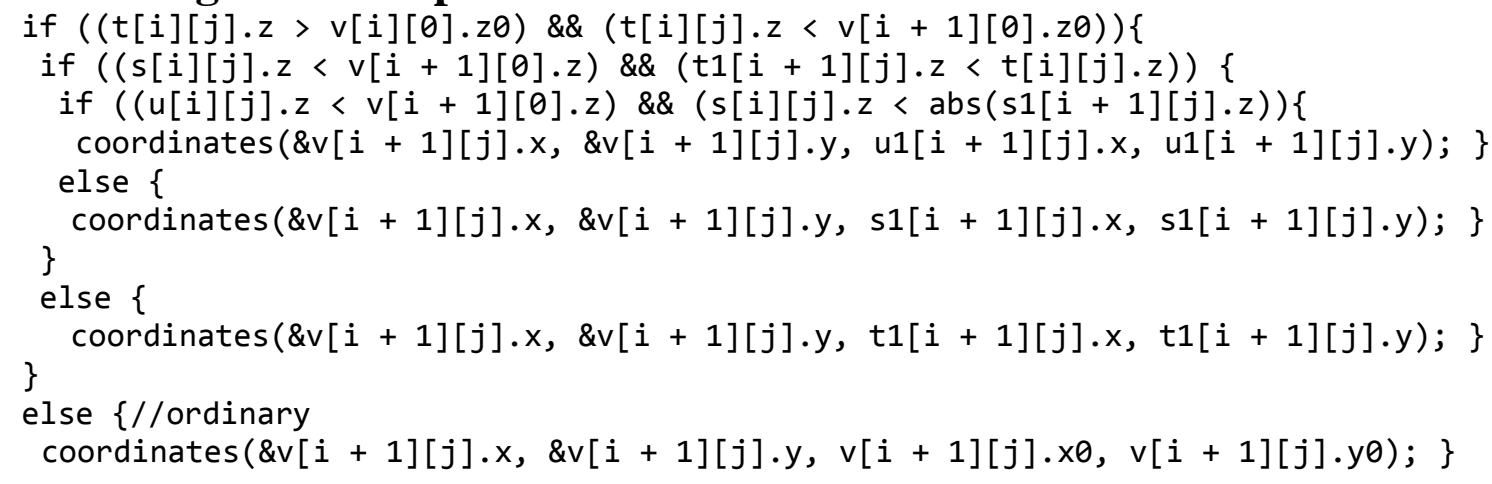

See the output screen in Fig. 15.

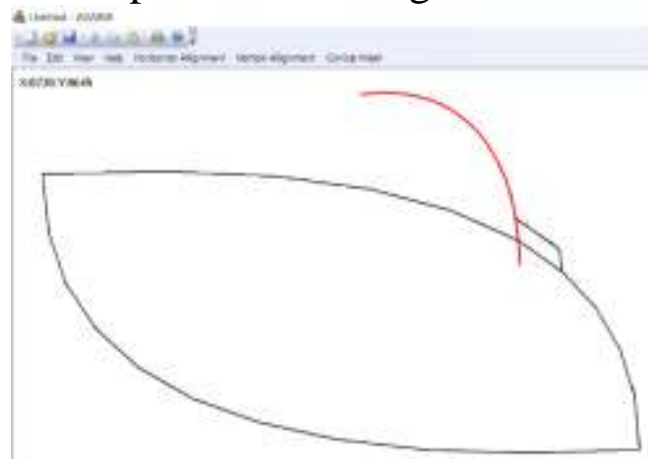

Fig. 16: An output screen for constructing the first face in the row.

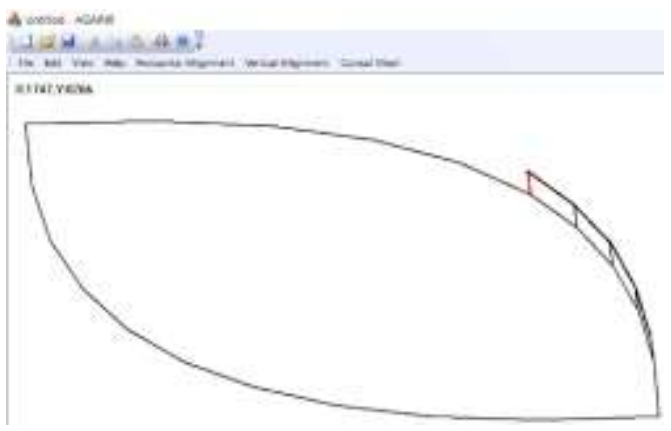

Fig. 17: An output screen for constructing the other faces in the row. 


\subsection{Starting Face Plane}

Let $\left\langle\Delta x_{0}, \Delta y_{0}, 0\right\rangle,\left\langle 0, \Delta y_{i}, \Delta z_{i}\right\rangle$ be direction vectors of $\overleftrightarrow{V_{l, 0}, V_{l, 1}}, r_{i, 0}$ respectively as shown in Fig. 11. $\therefore$ The equation of $\alpha_{i, 0}\left(V_{i, 0}, V_{i, 1}, V_{i+1,0}\right)$ is given by:

$E_{i, 0} x+F_{i, 0} y+G_{i, 0} z=H_{i, 0}$,

Where $E_{i, 0}=\Delta y_{0} \Delta z_{i}, F_{i, 0}=-\Delta x_{0} \Delta z_{i}, G_{i, 0}=\Delta x_{0} \Delta y_{i}$

$\theta_{i}=\Varangle\left(\alpha_{i, 0}, \pi_{i}\right)=\cos ^{-1}\left[\frac{G_{i, 0}}{\sqrt{\left(E_{i, 0}\right)^{2}+\left(F_{i, 0}\right)^{2}+\left(G_{i, 0}\right)^{2}}}\right]$

//constants of a plane defined by two straight lines

void plane(double x1, double x2, double y1, double y2, double z1, double z2, double d, double e, double f, double

$* \mathrm{E}$, double $* \mathrm{~F}$, double $* \mathrm{G}$, double $* \mathrm{H})\{$

$$
\begin{aligned}
& * \mathrm{E}=(\mathrm{y} 2-\mathrm{y} 1) * \mathrm{f}-(\mathrm{z} 2-\mathrm{z} 1) * \mathrm{e} ; \\
& * \mathrm{~F}=(\mathrm{z} 2-\mathrm{z} 1) * \mathrm{~d}-(\mathrm{x} 2-\mathrm{x} 1) * \mathrm{f} \\
& \left.*_{\mathrm{G}}=(\mathrm{x} 2-\mathrm{x} 1) * \mathrm{e}-(\mathrm{y} 2-\mathrm{y} 1) * \mathrm{~d} ;\right\}
\end{aligned}
$$

See the output screen in Fig. 16.

\subsection{General Face Plane}

Any other face of the row is defined by the plane $\alpha_{i, j} \supset \overleftrightarrow{V_{l, j}, V_{l, j+1}}: \Varangle\left(\alpha_{i, j}, \pi_{i}\right)=\theta_{i}$ as shown in Fig. 12. Let $\left\langle\Delta x_{i, j}, \Delta y_{i, j}, 0\right\rangle$ be a vector in the direction of $\overleftrightarrow{V_{l, j}, V_{l, j+1}}$ and $\langle A, B, C\rangle$ be a normal vector of $\alpha_{\mathrm{I}, \mathrm{j}}$. Based on the result of dot product of the orthogonal vectors, one can conclude that:

$$
A\left(\Delta x_{i, j}\right)+B\left(\Delta y_{i, j}\right)=0
$$

Since it is constrained that: $\Varangle\left(\alpha_{i, j}, \pi_{i}\right)=\theta_{i}$,

$\therefore \cos ^{2} \theta_{i}=\frac{C^{2}}{A^{2}+B^{2}+C^{2}} \quad$ i.e. $A^{2}+B^{2}=C^{2} \tan ^{2} \theta_{i}$

Solving $(a)$ and $(b)$ for the normal vector direction ratios:

$\frac{B}{C}=\frac{-\Delta x_{i, j} \tan \theta_{i}}{\sqrt{\left(\Delta y_{i, j}\right)^{2}+\left(\Delta x_{i, j}\right)^{2}}} \therefore \frac{A}{C}=\frac{\Delta y_{i, j} \tan \theta_{i}}{\sqrt{\left(\Delta y_{i, j}\right)^{2}+\left(\Delta x_{i, j}\right)^{2}}}$

$\therefore$ The equation of $\alpha_{i, j}$ is given by:

$E_{i, j} x+F_{i, j} y+G_{i, j} z=H_{i, j}$

Where $\left\{\begin{array}{c}E_{i, j}=\Delta y_{i, j} \\ F_{i, j}=-\Delta x_{i, j}\end{array}\left\{\begin{array}{c}G_{i, j}=\left(\cot \theta_{i}\right) \sqrt{\Delta x_{i, j}^{2}+\Delta y_{i, j}^{2}} \\ H_{i, j}=E_{i, j} x_{i, j}+F_{i, j} y_{i, j}+G_{i, j} z_{i, j}\end{array}\right.\right.$ 
//Other Planes of row j:

for (int $\mathrm{i}=1 ; \mathrm{i}<20 ; \mathrm{i}++)\{$

$\mathrm{p}[\mathrm{j}][\mathrm{i}] \cdot \mathrm{E}=\mathrm{v}[\mathrm{j}][\mathrm{i}+1] . \mathrm{y}-\mathrm{v}[\mathrm{j}][\mathrm{i}] . \mathrm{y}$

$\mathrm{p}[\mathrm{j}][\mathrm{i}] . \mathrm{F}=\mathrm{v}[\mathrm{j}][\mathrm{i}] . \mathrm{x}-\mathrm{v}[\mathrm{j}][\mathrm{i}+1] . \mathrm{x}$

p[j][i].G = pow $(\operatorname{pow}(p[j][i] . E, 2)+\operatorname{pow}(p[j][i] . F, 2), 0.5) / \tan$ Theta; $\}$

See the output screen in Fig. 17.

\subsection{Valence 4 Vertex}

The adjacent planes meridian segment $r_{i, j}\left(\alpha_{i, j-1} \cap \alpha_{i, j}\right)$ as shown in Fig. 13 has a direction vector of: $\left\langle E_{i, j-1}, F_{i, j-1}, G_{i, j-1}\right\rangle \times\left\langle E_{i, j}, F_{i, j}, G_{i, j}\right\rangle$.

Assuming $\left\{\begin{array}{l}a_{i, j}=F_{i, j-1} G_{i, j}-F_{i, j} G_{i, j-1} \\ b_{i, j}=E_{i, j} G_{i, j-1}-E_{i, j-1} G_{i, j} \\ c_{i, j}=E_{i, j-1} F_{i, j}-E_{i, j} F_{i, j-1}\end{array} \therefore r_{i, j} \equiv \frac{x-x_{i, j}}{a_{i, j}}=\frac{y-y_{i, j}}{b_{i, j}}=\frac{z-z_{i, j}}{c_{i, j}}\right.$

$\because V_{i+1, j}\left(x_{i+1, j}, y_{i+1, j}, z_{i+1, j}\right) \in r_{i, j}, z_{i+1, j}=z_{i+1,0}$.

$\therefore x_{i+1, j}=\frac{a_{i, j}}{c_{i, j}}\left(z_{i+1,0}-z_{i, j}\right)+x_{i, j}$

$y_{i+1, j}=\frac{b_{i, j}}{c_{i, j}}\left(z_{i+1,0}-z_{i, j}\right)+y_{i, j}$

\subsection{A valence 3 vertex:}

It is required to find the vertex $S_{0}\left(r_{i, j} \cap r_{i, j+1}\right)$ as shown in Fig. 14. The parametric equations of the two lines $r_{i, j}, r_{i, j+1}$ are given by:

$$
\left\{\begin{array}{l}
x=x_{i, j}+a_{i, j} t, y=y_{i, j}+b_{i, j} t, z=z_{i, j}+c_{i, j} t \\
x=x_{i, j+1}+a_{i, j+1} s, y=y_{i, j+1}+b_{i, j+1} s, z=z_{i, j+1}+c_{i, j+1} s \ldots \text { (b) }
\end{array}\right.
$$

at $S_{0}: x_{i, j}+a_{i, j} t_{T}=x_{i, j+1}+a_{i, j+1} s_{T} \ldots(c), y_{i, j}+b_{i, j} t_{T}=y_{i, j+1}+b_{i, j+1} s_{T} \ldots(d)$

Eliminating $\mathrm{s}_{\mathrm{T}}$ we get: $t_{T}=\frac{a_{i, j+1}\left(y_{i, j}-y_{i, j+1}\right)-b_{i, j+1}\left(x_{i, j}-x_{i, j+1}\right)}{a_{i, j} b_{i, j+1}-a_{i, j+1} b_{i, j}}$

Hence, coordinates of $T_{i, j}$ are given by: $\quad\left(x_{i, j}+a_{i, j} t_{T}, y_{i, j}+b_{i, j} t_{T}, z_{i, j}+c_{i, j} t_{T}\right)$

\section{Implemented Algorithm Results}

The following are some printed output of the program to show the variations of the constructed surfaces according to the horizontal and vertical alignment inputs.

Fig 18: Horizontal alignment consists of two parabolas. Vertical alignment is an elliptic segment.

Fig 19: Horizontal alignment consists of a parabola and a $4^{\text {th }}$ degree curve, vertical alignment is a circular arc.

Fig 20: The horizontal alignment is an ellipse, vertical alignment is a circular arc.

Fig 21: The horizontal alignment is a circle, vertical alignment is an elliptic segment. 

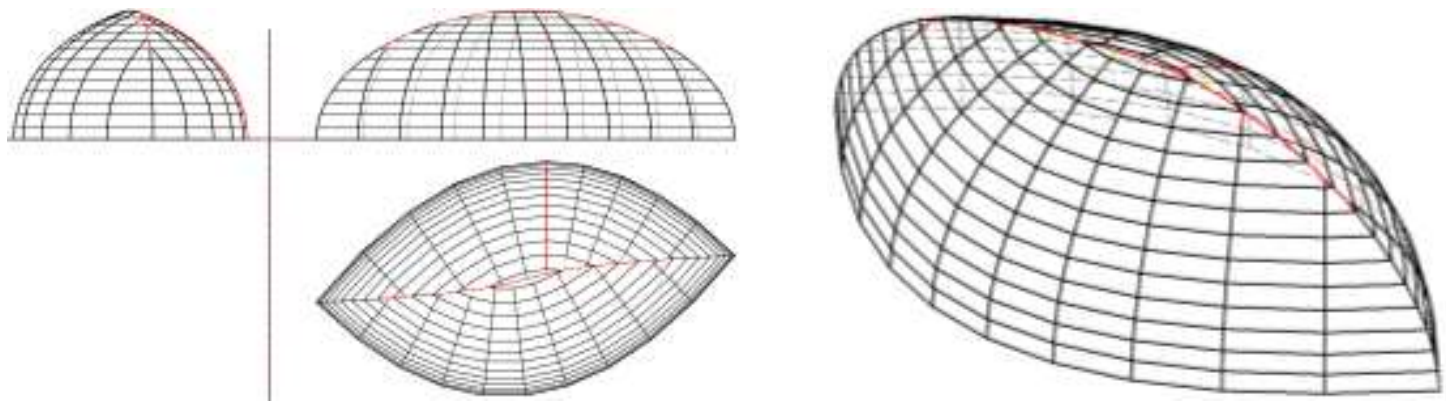

Fig. 18
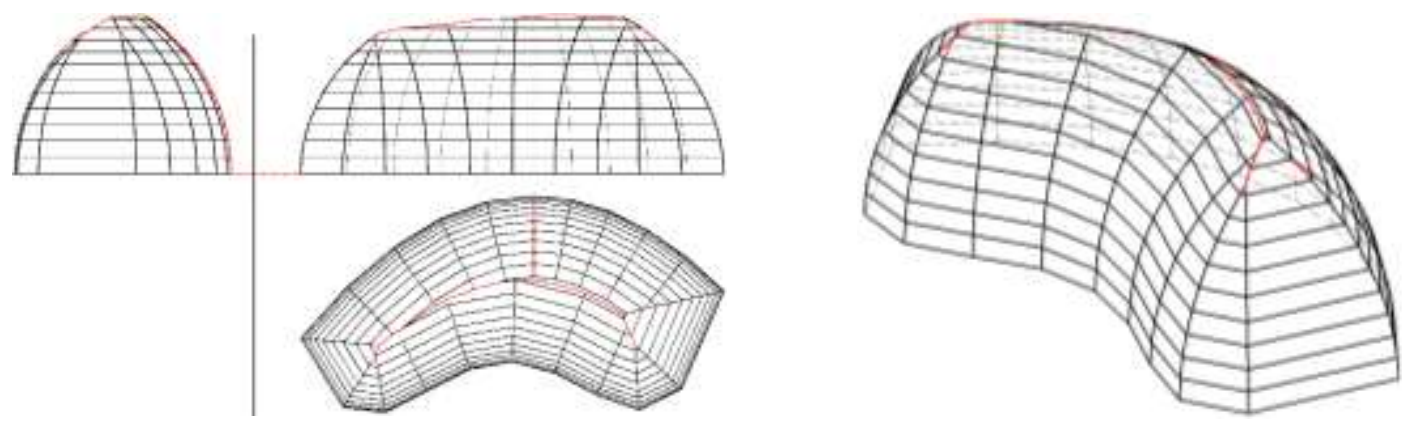

Fig. 19

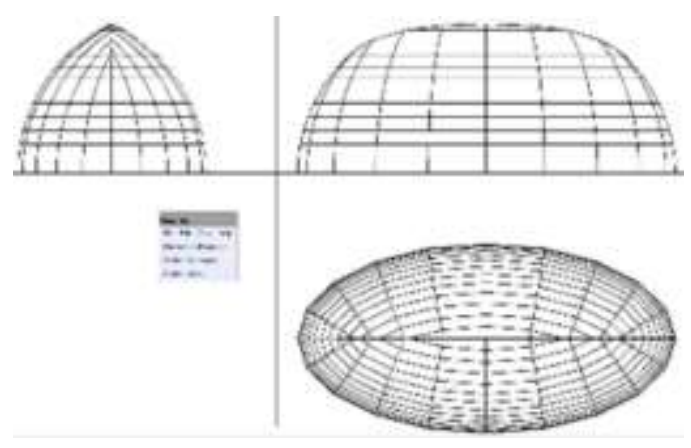

Fig 20

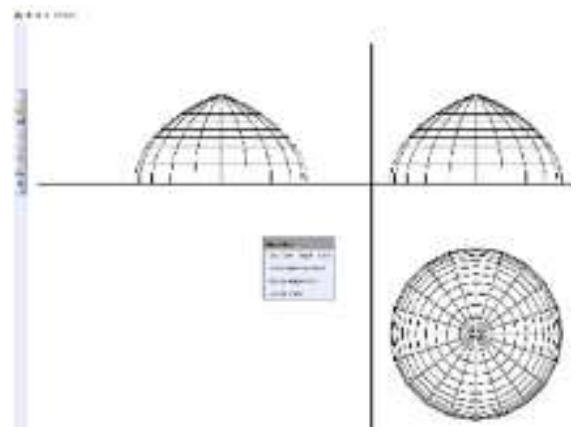

Fig 21

\section{Mesh Geometric Analysis}

\subsection{Meridian Plane}

Theorem (6): The plane $\beta_{i, j}$ bisecting $z\left(\alpha_{i, j-1}, \alpha_{i, j}\right)$ is vertical and is given by the equation of the horizontal projection of the line $r_{i, j}\left(\alpha_{i, j-1} \cap \alpha_{i, j}\right)$ and $\beta_{i, j} \equiv \mu_{j}$ where $\mu_{j}$ is the meridian plane.

Proof: Let $r_{j}^{\prime}$ be the horizontal projection of $r_{i, j}$ as shown in Fig 22 .

From the constants of (6):

$$
\sqrt{E_{i, j}^{2}+{F_{i, j}}^{2}+G_{i, j}^{2}}=G_{i, j} \sec \theta_{i}
$$


$\therefore \beta_{i, j}$ is given by: $\frac{E_{i, j} \boldsymbol{x}+F_{i, j} \boldsymbol{y}+G_{i, j} \mathbf{z}-H_{i, j}}{G_{i, j}}=\frac{E_{i, j-1} \boldsymbol{x}+F_{i, j-1} \boldsymbol{y}+G_{i, j-1} \mathbf{z}-H_{i, j-1}}{G_{i, j-1}}$

$\therefore\left(E_{i, j} G_{i, j-1}-E_{i, j-1} G_{i, j}\right) x-\left(F_{i, j-1} G_{i, j}-F_{i, j} G_{i, j-1}\right) y=H_{i, j} G_{i, j-1}-H_{i, j-1} G_{i, j}$ $=x_{i, j}\left[E_{i, j} G_{i, j-1}-E_{i, j-1} G_{i, j}\right]-y_{i, j}\left[F_{i, j-1} G_{i, j}-F_{i, j} G_{i, j-1}\right]$

$\therefore$ From constants of (7): $\beta_{i, j} \equiv \frac{\left(x-x_{i, j}\right)}{a_{i, j}}=\frac{\left(y-y_{i, j}\right)}{b_{i, j}} \equiv r_{i,}^{\prime}, \beta_{i, j}$ is a vertical plane $\because r_{i}^{\prime}$ is a horizontal edge view of the meridian. $\therefore \beta_{i, j} \equiv \mu_{j} \boldsymbol{\square}$.

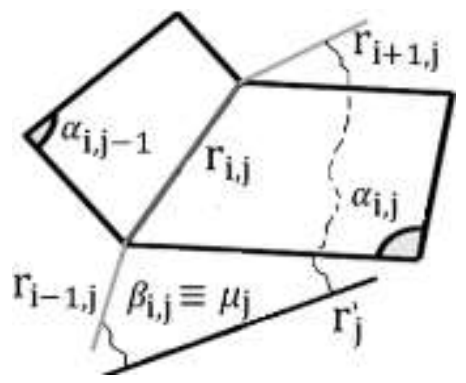

Fig. 22: The meridian plane $\mu_{i}$ bisects the angle between any two adjacent planes $\alpha_{i-1, j}$ and $\alpha_{i, j}$

\subsection{The Relation Between Semi Conical Angle and Semi Latitudinal}

\section{Angle}

Theorem (7): The semi conical angle $\delta_{i, j}=\Varangle\left(\alpha_{i, j}, \beta_{i, j}\right)$ is given by the relation:

$$
\cos \delta_{i, j}=\left(\cos \varepsilon_{i, j}\right)\left(\sin \theta_{i}\right)
$$

Where $\varepsilon_{i, j}$ is the semi latitudinal angle and $\theta_{i}$ is the inclination angle of the $i^{\text {th }}$ row planes.

Proof: For any two adjacent planes as shown in Fig. 23:

The vectors in the directions of $l_{i, j}$ and $r^{\prime}{ }_{j}$ are given by $\left\langle\Delta x_{i, j}, \Delta y_{i, j}\right\rangle$ and $\left\langle a_{i, j}, b_{i, j}\right\rangle$ :

$$
\cos \varepsilon_{i, j}=\frac{\left|a_{i, j} \Delta x_{i, j}+b_{i, j} \Delta y_{i, j}\right|}{\sqrt{a_{i, j}^{2}+b_{i, j}^{2}} \sqrt{\Delta x_{i, j}^{2}+\Delta y_{i, j}^{2}}}
$$

From the constants of $\alpha_{i, j}$ given by (6) and the constants of $\beta_{i, j}$ given by theorem 6 :

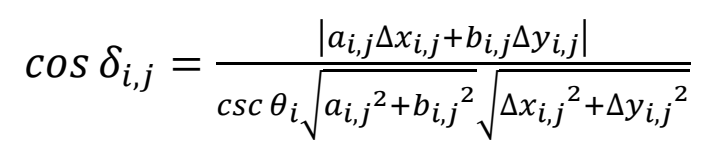

From $(a)$ and $(b): \cos \delta_{i, j}=\cos \varepsilon_{i, j} \sin \theta_{i}$ 


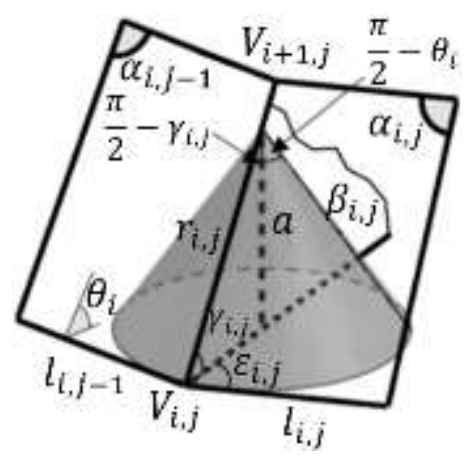

Fig. 23: The length of the meridian segment varies according to the angles $\theta_{i}$ and $\varepsilon_{i, j}$ and

\subsection{The Meridian Segment}

the vertical distance between $V_{i, j}$ and $V_{i+1, j}$

Theorem (8): The length $D_{i, j}$ of a meridian segment is given by:

$$
D_{i, j}=\left(z_{i+1,0}-z_{i, j}\right) \sqrt{\cot ^{2} \theta_{i} \csc ^{2} \varepsilon_{i, j}+1}
$$

Proof: Let $\delta_{i, j}=\Varangle\left(\alpha_{i, j}, \beta_{i, j}\right)$ and $\Gamma$ be a cone with vertical axis $a$, touching the planes $\alpha_{i, j-1}, \alpha_{i, j}$ as shown in Fig. 23, then its semi vertex angle is $\left(\frac{\pi}{2}-\theta_{i}\right)$ and $\Varangle\left(r_{i, j}, a\right)=\frac{\pi}{2}-\gamma_{i, j}$. From (4):

$$
\cos \delta_{i, j}=\left(\sin \theta_{i}\right) \sqrt{1-\left(\cot ^{2} \theta_{i}\right)\left(\tan ^{2} \gamma_{i, j}\right)} .
$$

From (12) and (14): $\cos \varepsilon_{i, j}=\sqrt{1-\left(\cot ^{2} \theta_{i}\right)\left(\tan ^{2} \gamma_{i, j}\right)}$

i.e. $\tan \gamma_{i, j}=\sin \varepsilon_{i, j} \tan \theta_{i}$

$$
\therefore \csc \gamma_{i, j}=\sqrt{\cot ^{2} \theta_{i} \csc ^{2} \varepsilon_{i, j}+1} \text {. }
$$

Using (9) and (10):

$$
D_{i, j}=\left(z_{i+1,0}-z_{i, 0}\right) \csc \gamma_{i, j} .
$$

Where $z_{i+1,0}$ and $z_{i, 0}$ are constants. From (15) and (16):

$$
D_{i, j}=\left(z_{i+1,0}-z_{i, 0}\right) \sqrt{\cot ^{2} \theta_{i} \csc ^{2} \varepsilon_{i, j}+1} \mathbf{m} .
$$

\subsection{Surfaces of Revolution}

Theorem (9): An input circular horizontal alignment results in a discrete analog of a surface of revolution.

Proof: For a row i of a conical mesh as shown in Fig. 24: $z_{i, 0}, z_{i+1,0}$ and $\theta_{i}$ are constants. As a circle is discretized by a regular polygon. $\therefore \varepsilon_{i, j}=\varepsilon_{i}$ for $j=$ $0,1, \ldots n-1 . \therefore$ From theorem (8) $\gamma_{i, j}=\gamma_{i}$ and $D_{i, j}=D_{i}$. Then, each lattitude $i+1$ 
is a discrete circle whose center lies above the center of lattitude $i$. Hence, each lattitude is a discrete circle whose center lies on the surface axis

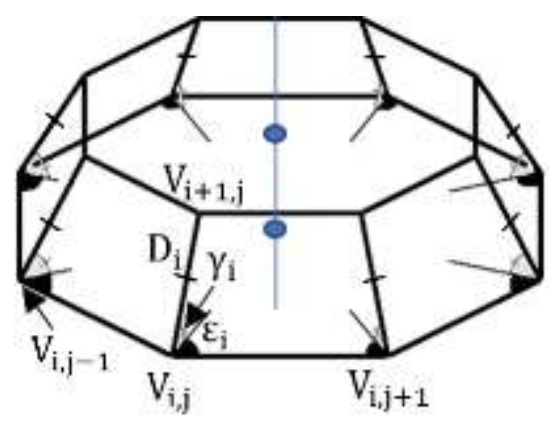

Fig. 24: One row of a discrete analog of a surface of revolution.

\section{Discussion}

The interpolation methods in section 3 were used in the implementation of the input horizontal and vertical alignments. Interpolation enables making variations in the geometric forms of input alignments. 3D Solid analytic geometry was used in the implementation of the required valence 4 dominant conical mesh. It is clear that the resulting surface varies according to the input horizontal and vertical alignments.

\section{Conclusion}

Our research work produced new implemented algorithms for constructing discrete analog of curves. They were used as inputs for the main algorithm of shape recognition with conical meshes, definitely as initial horizontal and vertical alignments for the produced surfaces. The resulting surface curvature varies according to the input initial horizontal and vertical alignments. Solid analytic geometry was used for deriving new theorems related to Architectural Geometry. The developed program has the ability to be developed for producing surfaces from more advanced initial horizontal and vertical alignments or from nonplanar curves. These surfaces are needed in both of architectural roofs and facades.

\section{References:}

[1] Liu, Y.; Pottmann, H.; Wallner, J.; Yang, Y. L.; Wang, W.; "Geometric modeling with conical meshes and developable surfaces"; ACM transactions on graphics TOG; Vol. 25, No. 3, pp. 681-689, 2006.

[2] Ross, E., Hambleton, D., \& Aish, R.; "Face-Offsetting Polygon Meshes with Variable Offset Rates"; Advances in Architectural Geometry 2016; pp. 40-61; 2016.

[3] S. Flöry; H. Pottmann; "Ruled Surfaces for Rationalization and Design in Architecture"; 2011

[4] Vaxman, A.; Müller, C.; Weber, O.; "Regular meshes from polygonal patterns". ACM Transactions on Graphics TOG, 36(4), 113, 2017.

[5] Wallner, J.; Pottmann, H.; "Geometric computing for freeform architecture." Journal of Mathematics in Industry 1.1 2011:

[6] Pottmann, H.; Wallner, J.; "Geometry and freeform architecture." Mathematics and Society: 131-151, 2016. 